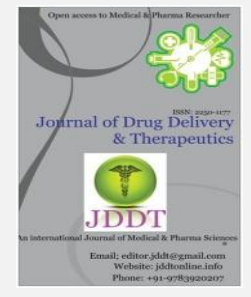

\title{
TRANSFEROSOME: A RECENT APPROACH FOR TRANSDERMAL DRUG DELIVERY
}

\author{
Abhay Kumar*, Amit Nayak, Sailesh Kumar Ghatuary, Sonali Dasgupta, Alok Pal Jain \\ Sarvepalli Radhakrishnan University, Bhopal (M.P.), India
}

\begin{abstract}
Novel drug delivery systems are now a day is creating a new interest in development of drug deliveries. The transdermal route of drug delivery has gained great interest of pharmaceutical research, as it circumvents number of problems associated with oral route of drug administration. Transferosomes are capable of transdermal delivery of low as well as high molecular weight drugs. This offers several potential advantages over conventional routes like avoidance of first pass metabolism, predictable and extended duration of activity, minimizing undesirable side effects, utility of short half life drugs, improving physiological and pharmacological response and have been applied to increases the efficiency of the material transfer across the intact skin, by the use of penetration enhancers and non-ionic surfactant vesicles. It is suitable for controlled and targeted drug delivery and it can accommodate drug molecules with wide range of solubility. Due to its high deformability it gives better penetration of intact vesicles. Transferosome possess an infrastructure consisting of hydrophobic and hydrophilic moieties together and as a result can accommodate drug molecules with wide range of solubility. They are biocompatible and biodegradable as they are made from natural phospholipids and have high entrapment efficiency. In this review, we have focused on transferosome with discussions on novel drug delivery systems for targeted delivery of therapeutics and important issues and challenges for future clinical applications.
\end{abstract}

Keywords: Novel drug delivery systems, Transferosomes, Transdermal drug delivery, Targeted drug delivery

Article Info: Received 12 Sep, 2018; Review Completed 07 Oct 2018; Accepted 08 Oct 2018; Available online 15 Oct 2018

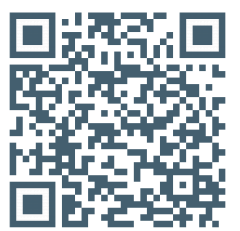

Cite this article as:

Kumar A, Nayak A, Ghatuary SK, Dasgupta S, Jain AP, Transferosome: A recent approach for transdermal drug delivery, Journal of Drug Delivery and Therapeutics. 2018; 8(5-s):100-104

DOI: http://dx.doi.org/10.22270/jddt.v8i5-s.1981

*Address for Correspondence:

Abhay Kumar, Sarvepalli Radhakrishnan University, Bhopal (M.P.), India

\section{INTRODUCTION}

Transfersome is a term registered as a trademark by the German company IDEA AG, and used by it to refer to its proprietary drug delivery technology. The name means "carrying body", and is derived from the Latin word 'transferre', meaning "to carry across", and the Greek word "soma", for a "body". A transfersome carrier is an artificial vesicle designed to be like a cell vesicle or a cell engaged in exocytosis, and thus suitable for controlled and, potentially targeted, drug delivery. Transferosomes are promising nanocarriers for non invasive transdermal delivery. Transferosomes are ultra deformable vesicles possessing an aqueous core surrounded by the complex lipid bilayer. Interdependency of local composition and shape of the bilayer makes the vesicle both self-regulating and selfoptimizing ${ }^{1}$. Transferosomes are capable of transdermal delivery of low as well as high molecular weight drugs ${ }^{2}$. Transferosomes are specially optimized, ultra flexible lipid supra molecular aggregates, which are able to penetrate the mammalian skin intact and then act as a drug carrier for non-invasive targeted drug delivery and sustained release of therapeutic agents ${ }^{3}$. Transferosomes are colloidal carriers which are easily accumulated into the leaky synovial tissue which leads to peripheral targeting. Transferosomes also act as depot resulting in controlled drug delivery system. Better drug delivery by transferosomes is due to the driving force provided by the osmotic gradient between outer and inner layer of stratum corneum ${ }^{4}$, thus, they can pass through the intact skin spontaneously under the influence of the naturally occurring in vivo transcutaneous hydration gradient. Due to their deformability, transferosomes are good candidates for the non-invasive delivery of small, medium, and large sized drugs. The transfersomes 
components that sustain strong membrane deformation preferentially accumulate, while the less adaptable molecules are diluted at sites of great stress. This dramatically lowers the energetic cost of membrane deformation and permits the resulting, highly flexible particles, first to enter and then to pass through the pores rapidly and efficiently. This behavior is not limited to one type of pore and has been observed in natural barriers such as in intact skin ${ }^{5,6}$. Transferosomes are self adaptable and optimized mixed lipid aggregate.
Transferosomes are artificial vesicles, being several orders of magnitude more deformable than standard liposomes. These are more elastic than standard liposomes. Transferosomes have been widely used as a novel carrier for effective transdermal drug delivery.

\section{MECHANISM OF ACTION}

Transferosomes overcome the skin penetration difficulty by squeezing themselves along the intracellular sealing lipids of stratum corneum (Figure 1).

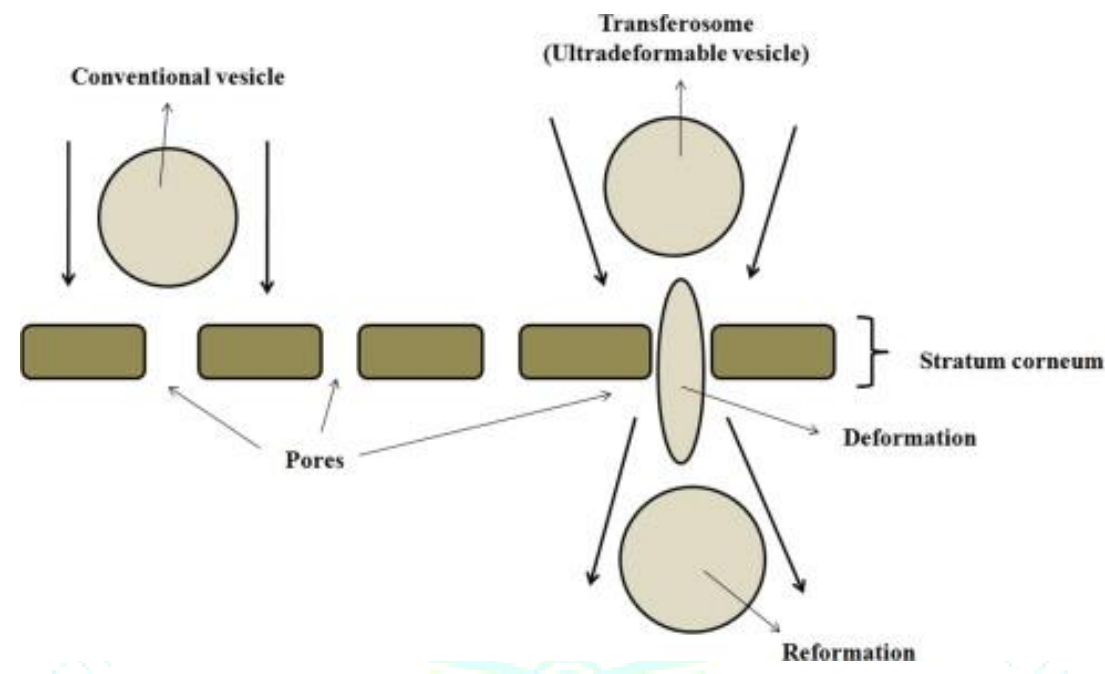

Figure 1: Schematic diagram describing interaction of the transferosome with skin tissue

At present, the mechanism of enhancing the delivery of active substances in and across the skin is not very well known. Two mechanisms of action have been proposed 7,8 . Transferosomes act as drug vectors, remaining intact after entering the skin. Transferosomes act as penetration enhancers, disrupting the highly organized intercellular lipids from stratum corneum, and therefore facilitating the drug molecules penetration in and across the stratum corneum. The recent studies propose that the penetration and permeation of the vesicles across the skin are due to the combination of the two mechanisms. Depending on the nature of the active substance (lipophilic or hydrophilic) and the composition of the transferosomes, one of the two mechanisms prevails.

After having penetrated through the outermost skin layers, transferosomes reach the deeper skin layer, the dermis. From this latter skin region they are normally washed out, via the lymph, into the blood circulation and through the latter throughout the body, if applied under suitable conditions. Transferosomes can thus reach all such body tissues that are accessible to the subcutaneously injected liposomes. The kinetics of action of an epicutaneously applied agent depends on the velocity of carrier penetration as well as on the speed of drug (re) distribution and the action after this passage. The most important single factors in this process are:

- Carrier in-flow

- Carrier accumulation at the targets site

- Carrier elimination
The onset of penetration-driving force depends on the volume of the suspension medium that must evaporate from the skin surface before the sufficiently strong trans-cutaneous chemical potential chemical potential or water activity gradient is established. Using less solvent is favourable in this respect. The rate of carrier passage across the skin is chiefly determined by the activation energy for the carrier deformation.

\section{COMPOSITION OF TRANSFEROSOMES}

Transfersomes are ultradeformable vesicle possessing an aqueous core surrounded by the complex lipid bilayer. Transferosomes possess an infrastructure consisting of hydrophobic and hydrophilic moieties together and as a result can accommodate drug molecules with wide range of solubility ${ }^{9}$. Transferosomes can deform and pass through narrow constriction (from 5 to 10 times less than their own diameter) without measurable loss. The transfersome is composed of two main aggregates namely. Firstly, an amphipathic ingredient (phosphatidylcholine), in which the aqueous solvents self-assembles into lipid bilayer that closes into a simple lipid vesicle. Secondly, a bilayer softening component (such as a biocompatible surfactant oramphiphile drug) that increases lipid bilayer flexibility and permeability. Transferosomes vesicles are composed of phospholipids as the main ingredient (soya phosphatidylcholine, egg phosphatidylcholine, dipalmityl phosphatidylcholine, etc), $10-25 \%$ surfactants for providing flexibility (sodium cholate, tween 80, span-80), 3-10\% alcohol as a solvent (ethanol, methanol) and hydrating medium consisting of saline phosphate buffer ( $\mathrm{pH}$ 6.5-7). 
They differ from liposomes because of the presence of so-called edge-activators, and comprise phospholipids as the main ingredient with $10-25 \%$ surfactant (e.g. sodium cholate) and 3-10\% ethanol. The surfactants are the "edge activators", which confer ultradeformability on the transferosomes. The elasticity of the vesicle is correlated with the quantity and the structure of the incorporated surfactant. In comparison with liposomes, it has been claimed that transferosomes are able to deliver their "payload" deeper into the skin.

\section{METHODS OF PREPARATION OF TRANSFERSOMES}

Transfersome vesicles are prepared in a similar manner as liposomes that includes sonicating, extrusion, low shear rates mixing (multilamellar liposomes), or high high-shear homogenisation unilamellar liposomes) of the crude vesicle suspension, except that no separation of the vesicle-associated and free drug is required. The preparation of transfersomes involves various process variables such as lecithin, surfactant ratio, effect of various solvents, effect of various surfactants and hydration medium. All the methods of preparation of transfersomes are comprised of two steps. First, a thin film is prepared hydrated and then brought to the desired size by sonication; and secondly, sonicated vesicles are homogenized by extrusion through a polycarbonate membrane (Figure 2). The mixture of vesicles forming ingredients, that is phospholipids and surfactant were dissolved in volatile organic solvent (chloroform methanol), organic solvent evaporated above the lipid transition temperature $\left(50{ }^{\circ} \mathrm{C}\right.$ for dipalmitoyl phosphatidyl choline) using rotary evaporator. Final traces of solvent were removed under vacuum for overnight. The deposited lipid films were hydrated with buffer ( $\mathrm{pH} 6.5$ ) by rotation at $60 \mathrm{rpm}$ min-1 fir $1 \mathrm{hr}$ at the corresponding temperature. The resulting vesicles were swollen for $2 \mathrm{hr}$ at room temperature. To prepare small vesicles, resulting $\mathrm{LMVs}$ were sonicated at room temperature or $50^{\circ} \mathrm{C}$ for $30 \mathrm{~min}$. using a B-12 FTZ bath sonicator or probe sonicated at $40 \mathrm{C}$ for $30 \mathrm{~min}$ (titanium micro tip, Heat Systems W 380). The sonicated vesicles were homogenized by manual extrusion 10 times through a sandwich of 200 and $100 \mathrm{~nm}$ polycarbonate membranes ${ }^{10}$.

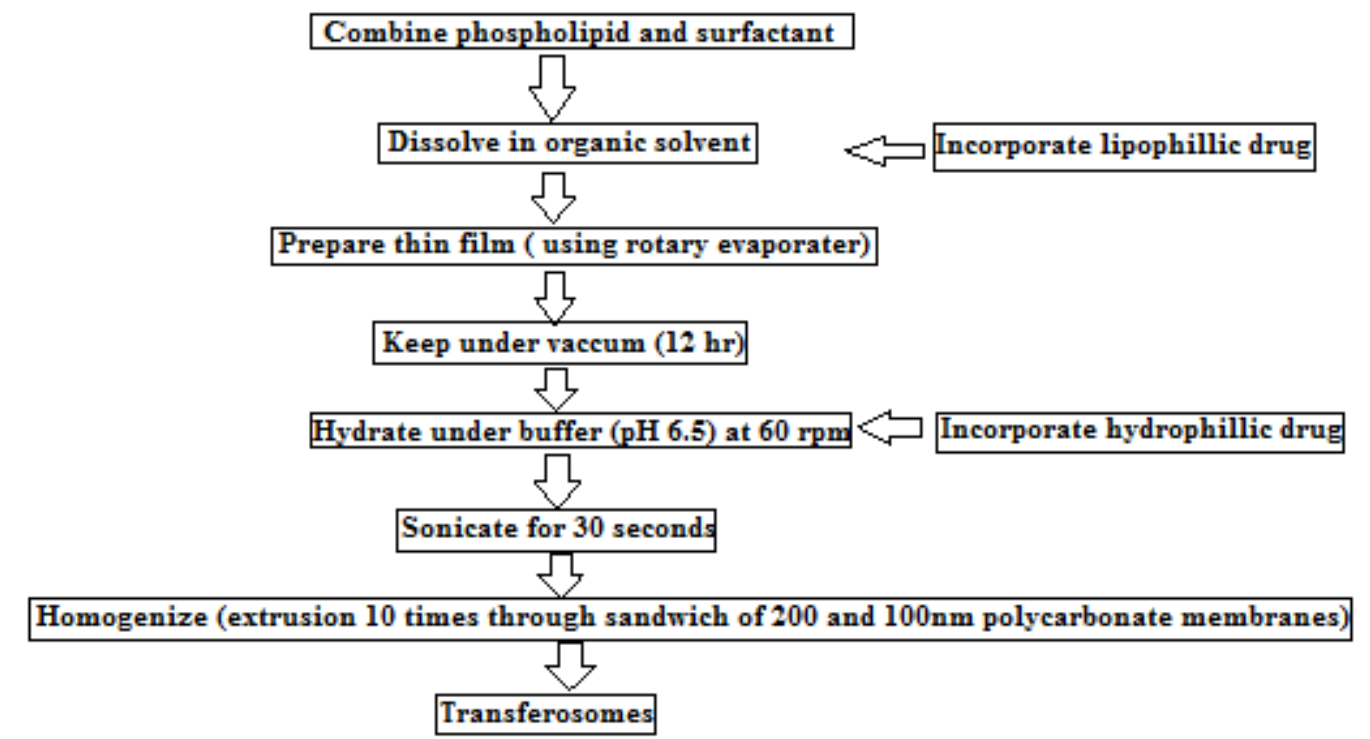

Figure 2: Method of preparation of transferosomes

\section{CHARACTERIZATION}

The mechanical properties and transport ability of a vesicle can be studied by measuring stress- or deformation-dependent vesicle bilayer elasticity and permeability changes. In a single experiment the objective may be reached by determining the pressure dependent area density of the transfersome suspension flux through a nano-porous filter, with pores at least $50 \%$ smaller than the average vesicle size. Analysis of experimental penetrability vs. driving pressure curves can yield the characteristic bilayer elasticity and permeability values, based on theoretical description of material flow as an activated transport process.

Visualization of transferosomes can be performed using transmission electron microscopy (TEM) and by scanning electron microscopy (SEM). Particle size and size distribution can be determined by dynamic light scattering (DLS) and photon correlation spectroscopy (PCS). The drug entrapment efficiency of transferosomes can be measured by the ultracentrifugation technique. Vesicle stability can be determined by assessing the size and structure of the vesicles over time and drug content can be quantified by HPLC or other spectrophotometric methods. In vitro drug release can be measured using a diffusion cell or a dialysis method ${ }^{9,11}$.

\section{APPLICATION OF TRANSFERSOMES}

They are used as a carrier for protein and peptides like insulin, bovine serum albumin, vaccines, etc. Because of their good penetration power and flexibility, transferosomes formulations are used for effective 
delivery of non-steroidal antiinflammatory agents like ibuprofen and diclofenac.

Insulin can be deliverd by encapsulating it into transferosomes. Insulin is generally administered by subcutaneous route that is inconvenient. Encapsulation of insulin into transferosomes (transfersulin) overcomes these entire problems. After transfersulin application on the intact skin, the first sign of systemic hypoglycemia are observed after 90 to $180 \mathrm{~min}$, depending on the specific carrier composition ${ }^{12}$. Transferosomes have been also used for the delivery of corticosteroids. Transferosomes improves the site specificity and overall drug safety of corticosteroid delivery into skin by optimizing the epicutaneously administered drug dose. Transferosomes based corticosteroids are biologically active at dose several times lower than the currently used formulation for the treatment of skin diseases ${ }^{13}$. Transfersomes have been widely used as a carrier for the transport of proteins and peptides. Proteins and peptide are large biogenic molecules which are very difficult to transport into the body, when given orally they are completely degraded in the GI tract. These are the reasons why these peptides and proteins still have to be introduced into the body through injections. Various approaches have been developed to improve these situations. The bioavaibility obtained from transferosomes is somewhat similar to that resulting from subcutaneous injection of the same protein suspension. The transferosomal preparations of this protein also induced strong immune response after the repeated epicutaneous application, for example the adjuvant immunogenic serum albumin in transferosomes, after several dermal challenges is as active immunologically as is the corresponding injected proteo-transferosomes preparations $14,15$. Transferosomes have also been used as a carrier for interferons, for example leukocytic derived interferone$\alpha$ (INF- $\alpha)$ is a naturally occurring protein having antiviral, antiproliferive and some immunomodulatory effects. Transferosomes as drug delivery systems have the potential for providing controlled release of the administered drug and increasing the stability of labile drugs. Hafer et al studied the formulation of interleukin2 and interferone- $\alpha$ containing transferosmes for potential transdermal application they reported delivery of IL-2 and INF- $\alpha$ trapped by transferosomes in sufficient concentration for immunotherapy ${ }^{16}$. Anti cancer drugs like methotrexate were tried for transdermal delivery using transfersome technology. The results were favorable. This provided a new approach for treatment especially of skin cancer 17,18 . Application of anesthetics in the suspension of highly deformable vesicles, transferosomes, induces a topical anesthesia, under appropriate conditions, with less than $10 \mathrm{~min}$. Maximum resulting pain insensitivity is nearly as strong $(80 \%)$ as that of a comparable subcutaneous bolus injection, but the effect of transferosomal anesthetics last longer [17]. NSAIDS are associated with number of GI side effects. These can be overcome by transdermal delivery using ultra-deformable vesicles. Studies have been carried out on diclofenac and ketoprofen. Ketoprofen in a transfersome formulation gained marketing approval by the Swiss regulatory agency (SwissMedic) in 2007; the product is expected to be marketed under the trademark diractin. Further therapeutic products based on the transfersome technology, according to IDEA AG, are in clinical development ${ }^{19}$. Transferosomes have also used for the delivery of corticosteroids. Transferosomes improves the site specificity and overall drug safety of corticosteroid delivery into skin by optimizing the epicutaneously administered drug dose. Transferosomes beased cortiosteroids are biologically active at dose several times lower than the currently usd formulation for the treatment of skin diseases ${ }^{20}$. Transfersomes have also been reported to improve the therapeutic efficacy of cyclosporine, and the site specificity and safety of corticosteroids. Transfersomes can penetrate stratum corneum and supply the nutrients locally to maintain its functions resulting maintenance of skin in this connection the transfersomes of capsaicin has been prepared by Xiao-Ying et al. which shows the better topical absorption in comparison to pure capsaicin ${ }^{21}$. Herbal drugs can also be incorporated into transferosomes as they can penetrate stratum corneum supply nutrients locally to maintain its functioning. curcumin, capsaicin showed topical administration through transferosomal formulations.

\section{LIMITATIONS OF TRANSFEROSOMES}

Transferosomes are chemically unstable because of their predisposition to oxidative degradation. Purity of natural phospholipids is another criteria militating against adoption of Transferosomes as drug delivery vehicles. Transferosomes formulations are expensive ${ }^{3,17,22}$.

\section{SCOPE OF TRANSFERSOMES}

Transfersome technology is best suited for noninvasive delivery of therapeutic molecules across open biological barriers. The transfersome vesicles can transport across the skin, for example, molecules that are too big to diffuse through the barrier. Examples include systemic delivery of therapeutically meaningful amounts of macromolecules, such as insulin or interferon, across intact mammalian skin. Other applications include the transport of small molecule drugs which have certain physicochemical properties which would otherwise prevent them from diffusing across the barrier. Another attraction of the transfersome technology is the carriers ability to target peripheral, subcutaneous tissue. This ability relies on minimization of the carrier associated drug clearance through cutaneous blood vessels plexus: the non-fenestrated blood capillary walls in the skin together with the tight junctions between endothelial cells preclude vesicles getting directly into blood, thus maximizing local drug retention and propensity to reach the peripheral tissue targets $[23,24$. Scope of transfersomes is mainly intended for topical application although other routes may be considered for further investigations. Drug should be selected in such a way that it fits in the criteria of topical delivery. It should have ideal limits for aqueous solubility, lipophilicity, molecular size, melting point and $\mathrm{pH}$ of the aqueous saturated solution. Further in future by combining various other strategies, vesicular system will find the central place in novel drug delivery, particularly in 
diseased cell sorting, diagnostics, gene and genetic materials, safe, targeted and effective in vivo delivery.

\section{CONCLUSION}

Transfersomes are the most promising transdermal drug carrier is the recently developed and patented Transfersome ${ }^{\circledR}$ which penetrates the skin barrier along the transcutaneous moisture gradient. This leads the carriers through the "virtual "pores between the cells in the organ without affecting its biological and general barrier properties. Transfersome carriers can create a highly concentrated drug depot in the systemic circulation. Transfersome carriers loaded with various

\section{REFERENCES}

1. Cevc G, Grbauer D, Schatzlein A, Blume G. Ultraflexible vesicles, transferosomes, have an extremely low pore penetration resistance ant transport therapeutic amounts of insulin across the intact mammalian skin. Biochem Biophys Act 1998; 201-215.

2. El-Maghraby GM, Williams AC. Vesicular systems for delivering conventional small organic molecules and larger macromolecules to and through human skin. Expert Opin Drug Deliv 2009; 149-163.

3. Walve JR, Bakliwal SR, Rane BR, Pawar SP. Transferosomes: A surrogated carrier for transdermal drug delivery system. Int J App Bio Pharm Tech. 2011; 2 (1):201214.

4. Kumar A, Pathak K, Bali V. Ultra-adaptable nanovesicular systems: a carrier for systemic delivery of therapeutic agents. Piroxicam Discovery Today 2012; 17(21- 22):1233-41

5. Bain KR., Hadgkraft AJ, James WJ, and Water KA: Prediction of percutaneous penetration STS Publishing, Cardiff 1993; 3b:226-234.

6. Cevc G, Blume G, Sehatzlein A, Gebauer D and Paul A: The skin- a pathway for systemic treatment with patches and lipid-based agent carriers Advance Drug Delivery Reviews 1996; 18:349- 378

7. Jain S., Jain P. Transferosomes: A novel vesicular carrier for enhanced transdermal delivery: development, characterization and performance evaluation, Drug Dev. Ind. Pharm. 2003; 29:1013-1026

8. Paul A., Cevc G., Bachhawat B.K. Transdermal immunization with an integral membrane component, gap junction protein, by means of ultradeformable drug carriers, transferosomes, Vaccine. 1998; 16 (2-3):188-195.

9. Jain NK. Advances in Controlled and Novel Drug Delivery. CBS Publishers and Distributers First edition. New Delhi. 2001; 426-451.

10. Fry DW, White JC and Goldman ID: Rapid separation of low molecular weight solutes from liposomes without dilution, Journal of Analytical Biochemistry 1978; 90:809-815

11. Touitou E., Godin B., Dayan N., Weiss C. Intracellular Delivery Mediated By An Ethosomal Carrier. Biomaterials. 2001; 22:3053-3059.

12. Gregor C, Dieter G, Juliane S, Andreas S, Gabriele B, "Ultra-flexible vesicles, Transfersomes, have an extremely agents of different molecular size and lipophilicity (lidocaine, tetracaine, cyclosporine, diclofenac, tamoxifen, etc.) have been shown to cross the skin barrier. In addition, polypeptides such as calcitonin, insulin, $\alpha$ - and $\gamma$ - interferon, and, $\mathrm{Cu}-\mathrm{Zn}$ super oxide dismutase, serum albumin, and dextrose have been successfully delivered across the skin with transfersome carriers. The future of controlled drug delivery is expected to grow phenomenonally and biomedical application of transfersomes is expected to increase and requires greater efforts towards investigating the nonbilayer phases and exploring the mechanism of action.

low pore penetration resistance and transport therapeutic amounts of insulin across the intact mammalian skin", Biophysica Acta, 1998, 1368:201-215.

13. Cevc G, "Isothermal lipid phase", Transitions Chemistry and Physics of Lipids, 1991, 57:293- 299.

14. Maghraby EI, Williams GM, Barry BW, "Skin delivery of oestradiol from lipid vesicles: importance of liposome structure", Int. J. Pharma, 2000, 204 (1-2):159-69.

15. Trotta M, Peira E, Carlotti ME, Gallarate M, "Deformable liposomes for dermal administration of methotrexate", Int. J. Pharma, 2004, 270:119.

16. Hafer C, Goble $\mathrm{R}$, Deering $\mathrm{P}$, Lehmer A, Breut J, "Formulation of interleukin-2 and interferon-alpha containing ultra-deformable carriers for potential transdermal application", Anticancer Res., 1999, 19 (2c), 1505-7.

17. Modi CD, Bharadia PD, "Transfersomes: New Dominants for Transdermal Drug Delivery", Am. J. PharmTech Res., 2012, 2 (3):71-91.

18. Benson HA, "Transfersomes for transdermal drug delivery", Expert Opin. Drug Deliv., 2006, 3 (6):727-37.

19. Dubey V, Mishra D, Asthana A, Jain NK, "Transdermal delivery of a pineal hormone: melatonin via elastic liposomes", Biomaterials, 2006, 27 (18):3491-6.

20. Cevc G, Blume G., Schatzlein A: Transfersomes-mediated transepidermal delivery improves the regiospecificity and biological activity of corticosteroids in vivo. Journal of Controlled Release 1997; 45:211- 226.

21. Wang XR, Gao SQ, Niu XQ, Li LJ, Ying XY, Hu ZJ, Gao JQ. Capsaicin-loaded nanolipoidal carriers for topical application: design, characterization, and in vitro/ in vivo evaluation. International Journal of Nanomedicine 2017; 12:3881-3898.

22. Kombath RV, Minumula SK, Sockalingam A, Subadhra S, Parre S, Reddy TR, David B, "Critical issues related to transfersomes - novel Vesicular system", Acta Sci. Pol., Technol. Aliment., 2012, 11 (1), 67-82.

23. Cho YW and Flynn M. Oral delivery of Insulin Wletterx, Lancet, 1989; 23-30: 1518-1519.

24. Wang PY. Implantable Reservoir for Supplemental Insulin Delivery on Demand by External Compression, Biomaterials, 1989; 10: 197-201. 